\title{
A randomized controlled trial of the effects of a prudent diet on cardiovascular risk factors, gene expression, and DNA methylation - the Diet and Genetic Intervention (DIGEST) Pilot study
}

Michael A. Zulyniak', Russell J. de Souza ${ }^{2,3,4}$, Andrew Mente ${ }^{5}$, Sujane Kandasamy², Monisha Nundy ${ }^{5}$, Dipika Desai ${ }^{1,5}$, Kripa Raman ${ }^{5}$, Ranya Hasso ${ }^{5}$, Guillaume Pare ${ }^{1,2,5}$, Joseph Beyene ${ }^{2}$ and Sonia S. Anand ${ }^{1,2,5^{*}}$

\begin{abstract}
Background: Risk of cardiovascular disease (CVD) can be increased by single-nucleotide polymorphisms (SNPs) in the 9p21 region of the genome. However, observational studies have shown that the deleterious effect of 9p21 SNPs on CVD might be offset by consuming a diet rich in fresh fruits and vegetables. This association may be driven by diet-influenced modifications in epigenetic and gene expression profiles. In this pilot study, we aimed to: $i$. test the feasibility of provision of a 'Prudent' and 'Western' diet outside of a specialized clinic, ii. assess the impact of each diet on cardiovascular risk factors.

Methods: A single centre, parallel two-arm, pilot randomized controlled trial (RCT) with food provision was conducted in a university teaching hospital outpatient clinic (McMaster university, Hamilton, ON, Canada). The aim was to recruit 80 participants, which allowed for a $10 \%$ dropout. The actual study consisted of 84 apparently healthy participants (69\% women, 18 to 77 years) at low cardiovascular risk. Participants were randomly assigned to follow one of two weight-maintaining diets: 'Prudent' or 'Western' for 2-weeks. The Prudent diet provided $<7 \%$ of energy as saturated fat, a polyunsaturated: saturated fat ratio of 1.0, and increased fibre, fruits and vegetables. The Western diet was high in processed foods and sugar, with a moderate quantity of fruit and vegetables. All food was provided. Feasibility outcomes included time to recruitment and retention of participants, successful provision of foods, along with adherence to and palatability of the assigned diets. Secondary outcomes were changes in total cholesterol, low density lipoprotein cholesterol (LDL-C), systolic blood pressure (SBP), and diastolic blood pressure (DBP) to obtain variance and estimates of effect sizes to inform the sample size calculation for a definitive trial.

(Continued on next page)
\end{abstract}

\footnotetext{
* Correspondence: anands@mcmaster.ca

${ }^{1}$ Department of Medicine, McMaster University, Hamilton, Ontario, Canada

${ }^{2}$ Department of Clinical Epidemiology and Biostatistics, McMaster University,

Hamilton, Ontario, Canada

Full list of author information is available at the end of the article
} 
(Continued from previous page)

Results: Recruitment was undertaken over 2 periods spanning 8 months between May-December, 2012. Response rates were high, with 228 screened participants yielding 84 randomized participants. Eight-two of 84 ( $98 \%$ ) of participants completed the intervention. Self-reported adherence to the diets was excellent ( $>92 \%$ of provided food consumed). The Prudent diet was $48 \%$ more palatable than the Western diet $(P<0.05)$. Participants receiving the Prudent diet showed a trend toward reduced systolic $(-4 \mathrm{mmHg} ; P=0.10)$ and diastolic $(-3 \mathrm{mmHg}$; $P=0.07)$ blood pressure, and total cholesterol $(-0.24 \mathrm{mmol} / \mathrm{L} ; P=0.08)$, compared to individuals receiving the Western diet. Data collection from all randomized participants was completed within 18 months.

Conclusions: Recruitment, and retention of apparently healthy, normotensive adults into a feeding study for a 2-week duration is feasible outside of specialized dietary clinic, and modest diet-related changes in biomarkers begin to appear after two weeks.

Trial registration: NCT01658137 (clinicaltrials.gov).

Keywords: Nutrition, Randomized controlled trial, Pilot study, Feasibility

\section{Background}

The majority of cardiovascular disease (CVD) is explained by conventional risk factors including cigarette smoking, abnormal lipid levels, high blood pressure, obesity, diabetes, and health behaviours including dietary intake, physical activity, and psychosocial stressors [1]. Genetic factors contribute to the development of these risk factors, and directly to CVD through other novel pathways. Since the advent of high throughput chip-based genotyping, more than 45 genetic variants have been found to be associated with myocardial infarction (MI) $[2,3]$.

The most robust genetic variant that has been consistently associated with MI and other forms of arterial disease is the 9p21 variant. This variant, located on chromosome 9, is common in the population, with $50 \%$ of people carrying one copy of the risk allele and an additional $25 \%$ of the population carrying two copies of the risk allele. Compared to individuals with no risk allele, the risk of MI with one copy of the risk allele is $15-20 \%$ higher, and the risk with two copies of the risk allele is $20-40 \%$ higher [4]. Further, the risk associated with two versus one risk allele is augmented $(+14 \%)$ in younger individuals $(<55 \mathrm{yrs})$ relative to older individuals [5]. To date the exact mechanism by which $9 \mathrm{p} 21$ variants increase the risk of $\mathrm{MI}$ is uncertain; however, $9 \mathrm{p} 21$ variants consistently appear to influence the expression of antisense non-coding RNA in the INK4 locus (ANRIL), which plays a role in regulating expression of cylcin-dependent kinase inhibitor $2 A$ (CDKN2A) and cylcin-dependent kinase inhibitor $2 B$ (CDKN2B), which may influence vascular integrity and atherogenesis $[6,7]$. Recently, we made the observation that among carriers of the risk allele, the associated risk of MI may be negated among individuals that report consuming a diet high in fruits and vegetables - suggesting a gene-diet interaction [8]. However, the mechanisms by which diet alters the genomic activity of $9 \mathrm{p} 21$ are not known, and the epigenetic responsiveness of the region to acute changes in diet has not been studied.
Improvements in total and low-density lipoprotein (LDL) cholesterol have been reported after 2- to 2.5weeks of increased vegetable, fruit, and nut consumption or increased polyunsaturated or monounsaturated fats in healthy men and women $[9,10]$. Major dietary factors that affect blood pressure (sodium, potassium, and dietary patterns) have each reduced BP within four weeks with the biggest drop typically seen after two weeks $[11,12]$. In preparation for a study to understand the genetic and epigenetic mechanisms underlying these epidemiologic findings, we enrolled two groups of individuals, young and older (i.e., < or $\geq 50$ years) into the Diet and Genetic Intervention (DIGEST) pilot study. In this report, we describe the study design, report on the feasibility of providing food to free-living participants outside of a specialty dietary clinic, and assess the magnitude of changes in markers of cardiovascular risk and inflammation that occur in response to a "Prudent" versus a "Western" diet administered to individuals $<$ or $\geq 50$ years of age after 2-weeks.

\section{Methods}

\section{Study design}

DIGEST is an investigator-initiated efficacy trial to understand the genetic and epigenetic mechanisms underlying the role of $9 \mathrm{p} 21$ genetic variants in modulating diet-associated cardiovascular risk. It is a parallel-2-arm randomized controlled trial with food provision to compare the effects on blood pressure (BP), inflammatory markers, lipids, gene expression and DNA methylation between a "Western" diet and a "Prudent" diet. The study took place at a university campus (McMaster University, Hamilton, $\mathrm{ON}$ ) and teaching hospital (McMaster University Medical Centre). Eligibility was determined through telephone screening. Eligible participants were randomized to one of two diets (Western or Prudent) and all food was provided for 2 weeks. The study was approved by the Hamilton Integrated Research Ethics Board (May 2012), and the protocol was registered with clinicaltrials.gov (NCT01658137). We 
have adhered to CONSORT guidelines (http://www.consortstatement.org/) for the reporting of clinical trials.

\section{Recruitment}

Participants were recruited through flyers posted on-campus, in-hospital, and at various places in close proximity to the University campus; as well as monthly runs of advertisements in a local newspaper (Hamilton Spectator, Hamilton, $\mathrm{ON})$. Recruitment was carried out by study coordinators in consultation with the study principal investigator (R.J.dS., S.S.A.) in two phases-the first phase (May-October 2012) targeted a population of generally healthy volunteers under the age of 50, and a second phase (October - December 2012) aimed at a population of generally healthy volunteers aged 50 or older. Enrolled participants were encouraged to refer spouses/partners, or other interested friends. In total, 228 participants inquired and were screened which yielded 84 randomized participants.

\section{Eligible participants}

Eligible participants were community-dwelling men and women willing to consume all foods in the Prudent and Western diets that were between the ages of 18 and 80 with a body-mass index (BMI) $<45 \mathrm{~kg} / \mathrm{m}^{2}$ and were free of cancer, renal and liver disease, and not taking medications for diabetes, hyperlipidemia, or hypertension. The full inclusion and exclusion criteria are presented in Table 1. Prior to enrolment, each participant read an abridged version of the study protocol and was provided an opportunity to ask questions of the study staff to ensure informed consent to participate in the study, including storage of blood, and genetic testing.

\section{Study protocol}

The study was a parallel-2-arm randomized controlled trial carried out between June 2012 and November 2013. Participants were seen at a screening visit 1- 2 weeks prior to beginning the dietary intervention. If eligibility criteria were met, subjects were randomized to either a "Prudent" low-saturated fat diet rich in fruits and vegetables, or a "Western" diet emphasizing processed foods. Each diet lasted for 2-weeks. All foods were provided. Body weight and percent body fat by bioelectrical impedance (Digital Weight Scale, tanita.com) were measured weekly, and whole blood samples were obtained at baseline and 2-weeks post-randomization. At each clinic visit, participant blood pressure was measured three times in the non-dominant arm using an automated mercury sphygmomanometer. Seven-day diet histories were obtained for the week prior to beginning the intervention. Completed menu checklists were returned after 1 and 2 weeks of treatment and checked by study personnel (M.N., S.K., R.J.dS.), who also recorded the participant's
Table 1 Inclusion and exclusion criteria

Inclusion criteria

- Willing and able to provide informed consent

- Willing and able to attend 3 clinic visits

- Willing and able to eat all foods on Prudent or Western diets

- Age $\geq 18$ years of age

Exclusion criteria (self reported)

- Alcoholic beverage intake >14 drinks/week

- Use of food or nutritional supplements that cannot be discontinued

- Current use of anti-inflammatory medications that cannot be discontinued

- Current use of anti-hypertensive medications that cannot be discontinued

- Current use of anti-cholesterol medications that cannot be discontinued

- Previous diagnosis of cardiovascular disease or cardiovascular event within past year

- Body-mass-index $\geq 45 \mathrm{~kg} / \mathrm{m}^{2}$

- High blood pressure (or on anti-hypertensive medications)

- Abnormal blood lipids (or on lipid-modifying medications)

- Diabetes mellitus (FPG $\geq 7.0$ ) or impaired fasting glucose (fasting

glucose 6.1 to 6.9)

- Renal insufficiency or disease

- Hepatitis or Jaundice

- Liver disease

- Tuberculosis

- HIV/AIDS

- Active malignancy or cancer treatment within past 1 year

- Inflammatory bowel disease, malabsorptive states

- Current asthma (use of bronchodilator $\geq 1$ time in past month)

- Any chronic disease likely to influence adherence to diet or participation in study

- Current or planned pregnancy during term of study

- Current use of oral contraceptives or hormone replacement therapy

previous week's exercise and ensured it was constant over the study period.

At each visit, participants recorded their overall feeling of satiety using a 9-point bipolar semantic scale in which -4 was excessively hungry, 0 was neutral, and +4 was uncomfortably full; gas and bloating on a 3-point scale in which -1 was less than usual, 0 was as usual, and +1 was more than usual; and palatability of the diet on a scale of 1 (not palatable) to 10 (highly palatable) [13]. Daily bowel habits were also recorded. Each week, participants were provided with a 7-day menu plan on which they checked off each item as eaten.

A simple randomization list, stratified by age $(<50$ and $\geq 50$ years) was created using a random number generator by a student who did not see participants. Opaque envelopes containing a treatment assignments were provided to study personnel responsible for providing food and seeing participants. Study personnel responsible for enrolling participants were not blinded to the participant's diet because they also purchased the food for each participant and reviewed the diet records at each visit. Staff responsible for the laboratory analysis were blinded to treatment and received samples labeled with bar codes.

\section{Participant compensation}

Participants were provided with parking vouchers (up to $\$ 20 \mathrm{CAD}$ ) to attend each of the screening, and on-study 
visits. All food was provided at no cost to the participants. No additional compensation was provided.

\section{Diets}

\section{Diet development}

Meal plans were designed by a registered dietitian and nutrition researcher (R.d S. and A.M.). Seven-day menu plans were provided to participants, who prepared their own meals with their own choices of low-caloric condiments/seasonings (e.g. ketchup, salt, pepper, spices, etc.). Cooking fats (e.g. plant oils and butter) were provided and encouraged as per diet assignment. Representative menu plans for the Western and prudent diets are shown in Additional file 1: Table S1.

The "Prudent" diet was based on the DASH diet, which encourages vegetables, fruits, whole grains, fat-free or low-fat dairy products, fish, poultry, beans, nuts, and vegetable oils; and limited foods high in saturated fat ( such as fatty meats, full-fat dairy products, and tropical oils such as coconut, palm kernel, and palm oils) and sugar-sweetened beverages and sweets [14]. The Prudent diet achieved a low saturated fat content $(<7 \%)$ by including small amounts of lean animal products, low-fat dairy, plant proteins, and maintained a polyunsaturated:saturated fat ratio (P:S) of 1.0 by use of unsaturated vegetable oils. At least 6 cup equivalents of fruits and vegetables per day were provided; and overall, the diet provided $40 \mathrm{~g}$ fiber per 2000 kilocalories (kcal) from whole grains, nuts, fruits and vegetables.

The "Western" diet was characterized by a higher intake of red and processed meat, butter, high-fat dairy products, eggs, refined grains, processed potato products (such as french fries and hash browns), and high-sugar drinks and sweets [15]. This diet achieved a high saturated fat $(\approx 13 \%)$ content with a P:S ratio of 0.5 by providing fats as butter, in ground beef products, fullfat dairy/ice cream/cheese; and was kept low in dietary fibre $(<14 \mathrm{~g} / 2000 \mathrm{kcal})$, by providing refined grains, and $<3$ cup equivalents of fruits and vegetable/d mainly from juices.

\section{Energy balance}

All diets were designed to be weight-maintaining; and 7-day meal plans were developed and provided to participants at levels of 1600, 1800, 2100, 2300, 2500, 2700, 3000, and $3200 \mathrm{kcal} / \mathrm{d}$. Energy requirements were estimated at the screening visit using the HarrisBenedict equation, multiplied by an appropriate activity factor for each individual [16]. Energy requirements were reassessed at the week 1 visit based on measured weight change from baseline and diets were adjusted by adding or reducing the amount of foods to achieve weight stability.

\section{Food provision}

All foods (except coffee, tea, and condiments) were purchased from a local supermarket (Fortino's, Hamilton, $\mathrm{ON}$ ) and provided to participants. The first hamper was provided at the randomization visit (week 0), and a "top up" as needed at week 1 . Because our outpatient clinic does not have cold storage facilities for food, participants obtained their foods in one of three ways: 1) home delivery, in which a member of the research team or student volunteer purchased the foods and delivered them to the participant's home at a mutually-convenient time; 2) instore pick-up, in which a member of the research team or student volunteer purchased the foods from a local supermarket and the participant met the staff member at the store to pick up the foods; or 3) in-clinic pick-up, in which a member of the research team or student volunteer purchased the food in the hour preceding the participant's study visit, and provided food to the participant at his/her clinic visit.

\section{Lipid and inflammatory marker analysis}

Fasting serum samples were collected at weeks 0 and 2, and stored in liquid nitrogen $\left(-160{ }^{\circ} \mathrm{C}\right)$ until the end of study, when they were analyzed for inflammatory and lipid markers at the Clinical Research and Clinical Trials Laboratory (CRCTL; Population Health Research Institute, Hamilton, $\mathrm{ON}$ ). Total- and high-density lipoprotein-cholesterol (TC and HDL-C), and triglycerides (TG) were measured by timed end-point method under standard laboratory protocols using the Beckman Coulter Unicel ${ }^{\circ}$ DxC600 Synchron ${ }^{\circ}$ Clinical System and reagents (Beckman, California); non-HDL-C was calculated as TC minus HDL-C.

\section{Additional sample collection}

Participants consented to having additional fasting venous whole blood samples drawn and provided spot urine samples for future genetic, epigenetic, and metabolomic studies. A spot urine has been shown to provide accurate measures of sodium and potassium intake during the previous 24-hours when validated against 24-hour urine collections. Four $1 \mathrm{~mL}$ aliquots each of blood and urine were stored in $-70{ }^{\circ} \mathrm{C}$ freezers at CRCTL.

\section{Statistical analyses}

Results in text are presented as mean (SD) unless otherwise noted. Where values were measured at both baseline and follow-up, the primary method of analysis for between-treatments comparisons for all continuous outcomes was an analysis of covariance, with the week 2 value as the outcome variable, diet assignment as the independent variable (main effect), and baseline value, age, and sex as covariates to reduce the impact of confounding by imbalance in these factors between randomized groups. A 2-tailed paired t-test was used to assess the significance 
of within-treatment unadjusted changes from baseline. Means of continuous variables which were not measured at baseline (i.e. adherence, or intervention dietary intake) were compared with independent samples t-test. The unstandarized beta-coefficient from linear regression models were used to describe the association between changes in diet and changes in outcome variables where stated. The significance of between-treatment differences in proportions of categorical variables, i.e. symptom reports, was assessed with Fisher's exact test. In order to avoid type-2 errors at this exploratory stage, we did not correct for multiple testing. For this pilot study, statistical tests yielding a nominal $P<0.05$ were considered statistically significant; and those at $0.05<\mathrm{P}<0.10$ are reported as "trends". Analyses of adherence, anthropometric, symptom and biomarker data were conducted using linear models in SAS version 9.4 (SAS Institute, Cary, NC, USA).

\section{Power and sample size considerations}

The secondary aim of this study was to obtain data on changes in genetic and epigenetic marks and cardiovascular risk factors in response to a diet intervention to aid in the design of a future definitive trial $[9,10]$.

\section{Results}

Feasibility

\section{Recruitment and retention}

In total, 228 participants were telephone screened, yielding 84 enrolled adults (58 women and 26 men) with a mean age of 43.9 years (range: 18 to 77 years) and BMI of $25.6 \mathrm{~kg} / \mathrm{m}^{2}$ (range 19.3 to $44.8 \mathrm{~kg} / \mathrm{m}^{2}$ ). We were required to screen approximately 3 interested participants per successful enrolment. Baseline demographic characteristics, cardiovascular risk factors (Table 2) and diet profiles (Table 3) of these participants were comparable between the two randomized arms, with the exception that men assigned to the Western diet had higher nonHDL-C (3.95 [0.73] vs. 2.98 [0.75] $\mathrm{mmol} / \mathrm{L} ; P<0.003$; Table 2). Two participants, one from each diet assignment withdrew during the first week of the study due to difficulties following the assigned diet. Eighty-two of 84 participants completed the study (98 \%) (Fig. 1).

\section{Adherence}

For the majority of participants, adherence was excellent as assessed from completed diet checklists. 74 of 84 participants $(88.1 \%)$ returned a completed 7-day food record at baseline, and both food checklists at weeks 1 and 2 . When expressed as the percentage of prescribed calories recorded as eaten, adherence was $99.7 \%$ on the Western diet and $92.9 \%$ on the Prudent diet, according to self-reported checklists (Table 4). The lower percentage of provided foods reported as consumed on the Prudent
Table 2 Baseline characteristics of participants by diet assignment

\begin{tabular}{|c|c|c|c|}
\hline & Prudent $(n=46)$ & Western $(n=38)$ & $P$-value \\
\hline \multicolumn{4}{|l|}{$\overline{\operatorname{Sex}, n(\%)^{a}}$} \\
\hline Male & $14(30.4)$ & $12(31.6)$ & 1.00 \\
\hline Female & $32(69.6)$ & $26(68.4)$ & \\
\hline \multicolumn{4}{|l|}{ Ethnicity, $\mathrm{n}(\%)^{\mathrm{a}}$} \\
\hline White & $34(73.1)$ & $30(79.0)$ & 0.32 \\
\hline Black/African Canadian & $2(4.4)$ & $0(0.0)$ & \\
\hline Asian & $8(17.3)$ & $8(21.1)$ & \\
\hline Other & $2(4.4)$ & $0(0.0)$ & \\
\hline Age, mean $(S D)^{b}$ & $44.1(19.9)$ & $43.6(19.1)$ & 0.92 \\
\hline Body weight $(\mathrm{kg})^{\mathrm{b}}$ & $72.4(16.3)$ & $69.2(13.2)$ & 0.32 \\
\hline Height $(\mathrm{cm})^{\mathrm{b}}$ & $166.7(8.9)$ & $166.3(9.4)$ & 0.80 \\
\hline Body-mass index $\left(\mathrm{kg} / \mathrm{m}^{2}\right)^{\mathrm{b}}$ & $26.1(5.6)$ & $25.1(4.5)$ & 0.37 \\
\hline Percent body fat & $27.9(9.0)$ & $28.6(11.7)$ & 0.72 \\
\hline Waist circumference & $80.8(13.6)$ & $78.0(12.4)$ & 0.34 \\
\hline Women & $79.2(14.9)$ & $74.2(11.2)$ & 0.17 \\
\hline Men & $84.8(10.1)$ & $86.6(11.3)$ & 0.67 \\
\hline \multicolumn{4}{|l|}{ Blood pressure $(\mathrm{mmHg})$} \\
\hline Systolic ${ }^{b}$ & $117.6(17.1)$ & $113.1(17.9)$ & 0.25 \\
\hline Diastolic ${ }^{b}$ & $74.2(10.5)$ & $73.9(9.7)$ & 0.87 \\
\hline \multicolumn{4}{|l|}{ Lipids (mmol/L) } \\
\hline Total Cholesterol $^{\mathrm{b}}$ & $4.98(1.31)$ & $5.30(1.07)$ & 0.23 \\
\hline $\mathrm{HDL}-\mathrm{C}^{\mathrm{b}}$ & $1.50(0.40)$ & $1.47(0.41)$ & 0.72 \\
\hline Women & $1.55(0.36)$ & $1.56(0.40)$ & 0.97 \\
\hline Men & $1.35(0.44)$ & $1.23(0.26)$ & 0.40 \\
\hline Non-HDL-C ${ }^{b}$ & $3.48(1.17)$ & $3.83(0.94)$ & 0.14 \\
\hline Women & $3.64(1.24)$ & $3.73(1.03)$ & 0.76 \\
\hline Men & $2.98(0.75)$ & $3.95(0.73)$ & 0.003 \\
\hline
\end{tabular}

diet amounted to a net $157 \mathrm{kcal} / \mathrm{d}$ deficit compared with the Western diet $(P=0.042)$, but this did not result in a significantly different change in body weight over the 2-weeks. Those assigned to the Prudent diet reported $\approx 7$-cup equivalent servings of fruits and vegetables per 2,000 kcal/ day, with higher intakes of protein, carbohydrate, dietary fibre, vitamin $\mathrm{C}$, potassium, and magnesium; and lower intakes of total and saturated fat, dietary cholesterol, and sodium than those assigned to the Western diet, who reported $<2$ cup equivalent servings of fruits and vegetables per $2000 \mathrm{kcal} /$ day (Table 4). Most participants made use of at least two of three food provision options (i.e. delivery, instore pick-up, or clinic pick-up) during the study.

\section{Symptoms and palatability}

The mean palatability score was higher for the Prudent than the Western diet $(6.9 \pm 1.4$ vs $4.7 \pm 1.6$ out of 10 ; $P<0.001$ ), but neither diet was reported to be more 
Table 3 Baseline nutrient intake of participants (mean of 7-day diet histories) by diet assignment

\begin{tabular}{lll}
\hline Nutrient & Prudent $(n=40)$ & Western $(n=34)$ \\
\hline Energy (kcal/d) & $2030(546)$ & $1872(508)$ \\
Total protein (\% energy) & $17.2(3.2)$ & $17.9(3.3)$ \\
Available carbohydrate (\% energy) & $46.6(8.6)$ & $47.6(5.9)$ \\
Total dietary fiber (g/2000 kcal) & $21.9(7.4)$ & $23.9(7.8)$ \\
Total fat (\% energy) & $33.8(6.3)$ & $31.3(4.3)^{\dagger}$ \\
Saturated (\% energy) & $11.6(2.9)$ & $10.7(2.5)$ \\
Polyunsaturated (\% energy) & $5.2(2.0)$ & $5.0(1.4)$ \\
Monounsaturated (\% energy) & $10.4(4.0)$ & $9.2(2.6)$ \\
P:S ratio & $0.47(0.19)$ & $0.49(0.18)$ \\
Dietary cholesterol (mg/2000 kcal) & $306(111)$ & $334(142)$ \\
Alcohol (\% energy) & $2.8(3.3)$ & $2.0(3.1)$ \\
Vitamin C (mg/2000 kcal) & $93(52)$ & $102(46)$ \\
Potassium (mg/2000 kcal) & $2455(609)$ & $2529(694)$ \\
Magnesium (mg/2000 kcal) & $253(74)$ & $266(72)$ \\
Sodium (mg/2000 kcal) & $2584(605)$ & $2705(656)$ \\
Vegetables (cup eq./2000 kcal) & $1.6(1.0)$ & $1.8(0.9)$ \\
Fruits (cup eq./2000 kcal) & $1.4(0.9)$ & $1.4(0.9)$ \\
Fruits \& Veg (cup eq./2000 kcal) & $3.1(1.4)$ & $3.2(1.3)$ \\
\hline
\end{tabular}

Data are presented as means (SD). Significant between-diet differences are denoted by $\dagger$ at $P<0.05$ (independent samples t-test)

palatable than the participants' pre-study diet. Detailed symptom diaries were completed by 74 of 84 participants $(88.1 \%)$ at 1 or 2 weeks post-intervention (Table 5). Five of 35 participants (14.3\%) on the Western and two of 38 participants $(5.3 \%)$ on the Prudent diet indicated hunger ( $P=0.25$ for between-diets difference), while 2 participants on the Western diet (2.9\%) reported being uncomfortably full. Nineteen participants (59.4\%) on the Western and eleven participants $(32.4 \%)$ on the Prudent diet indicated more gas and/or bloating than usual $(P=0.047$ for between-diets difference). During week 1 , those assigned to the Western diet were more likely to report at least 1 day per week with constipation (12 of 37 [32.4\%]) than those assigned to the Prudent diet (4 of 45 [8.9\%]). During week 2 , those assigned to the Western diet were also more likely to report at least 1 day per week with constipation (9 of 37 [24.3\%]) than those assigned to Prudent (1 of 45 [2.2\%]; $P$-value for between-diet differences $\leq 0.01$ at both time points) but there were no changes in frequency of bowel movements from baseline in either diet.

\section{Participant feedback}

At each visit, participants were asked to verbalize their experiences with the diet during the previous week, with respect to ease of adherence, overall satisfaction, and barriers to adherence. After the first week, 35 participants assigned to the Western diet provided 86 comments; and 44 participants assigned to the Prudent diet provided 73 comments (Additional file 2: Table S3).

The most common negative experiences on the Western diet related to adverse gastrointestinal symptoms such as constipation, bloating, diarrhea, cramping or heartburn (mentioned in $26.7 \%$ of comments); feeling of poor health, low in "energy", or lacking mental focus (16.3\%). The diet itself was described as "not very palatable" (9.3\%), with "not enough variety" (7.0\%), and "difficult to stick to" (5.8\%). At the 2-week visit, $45 \%$ of the participants felt that their adherence and satisfaction with the diet had improved since the week- 1 visit, with $26 \%$ feeling it was the same, and $7 \%$ noted it was worse. Provision of less food and more fruits and vegetables were suggestions participants made to improve the diet.

The most common negative pieces of feedback received about the Prudent diet were that "too much food was provided" (17.8\%), "preparation was time-consuming" (12.3\%), and some negative gastrointestinal symptoms, such as bloating and constipation (8.2 \%). Positive feedback provided about the Prudent diet was that participants felt "healthier", "full of energy", and "less tired" (17.8\%), and experienced better gastrointestinal function, with easier and more frequent bowel movements $(8.2 \%)$. At the 2 -week visit, $48 \%$ of the participants felt that their own adherence and satisfaction with the diet improved after the first week, with $39 \%$ feeling it was the same, and $14 \%$ feeling it was worse. Suggestions provided by participants to improve the Prudent diet were to provide more detailed recipes, suggest more flavourful dishes, and reduce the volume of food provided.

\section{Diet modifications during the study}

To improve adherence during the study, small modifications were made to the diet plans for individuals as necessary. At baseline, one participant requested to substitute fish products with other foods, and this was accommodated by replacing fish with chicken and lean deli meats. Three participants $(8.5 \%)$ on the Western arm, and three participants $(6.8 \%)$ on the Prudent arm required decreases in energy levels to improve adherence or maintain weight; one participant $(2.2 \%)$ on the Prudent diet requested an increase in food to maintain satiety and weight. Minor requests for changes in the control diet that were accommodated included drinking less milk $(n=1)$, having fewer hamburger patties $(n=1)$, and switching some of the carbonated soft drinks for fruit drinks $(n=1)$. A request to eat a lean turkey submarine sandwich on whole wheat bread instead of the prescribed deli meat sandwich on whole wheat bread $(n=1)$ was accommodated on the Prudent arm. 


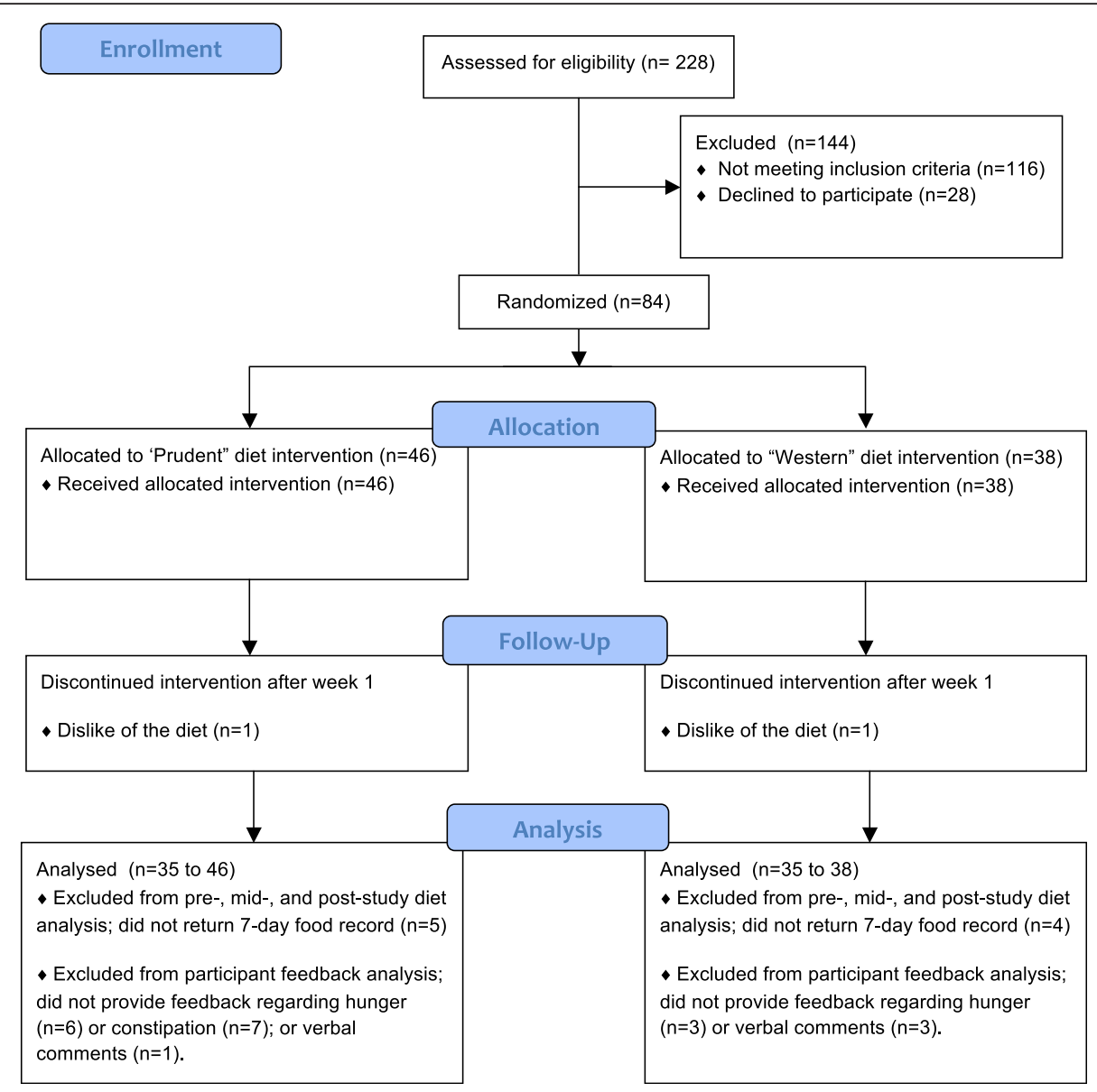

Fig. 1 CONSORT Flow Diagram

\section{Body weight}

Participants in both groups lost small yet statistically significant amounts of weight (Prudent, $0.6 \mathrm{~kg} ; P<0.01$ vs. Western, $0.4 \mathrm{~kg} ; P=0.03)$, but between-treatment differences were not significant $(P=0.62)$. Percent body fat was unchanged after 2 weeks on the Western Diet, but fell by $1.3 \%$ $(P<0.01)$ on the Prudent diet, yielding a significant between-treatment difference of $-1.5 \%(P=0.04)$.

\section{Changes in serum lipids and blood pressure}

The percent changes from baseline to week 2 in total cholesterol were as follows: In the prudent diet group, total cholesterol decreased by $3.9 \%(P=0.05)$, whereas in the Western diet group, total cholesterol did not decline substantially $(+0.6 \% ; P=0.69)$ (Table 6$)$. Change in total cholesterol over 2 weeks on the Prudent diet was related to the baseline total cholesterol level $(\beta=-0.143$; $P=0.06)$, but no such association was seen in the Western $\operatorname{diet}(\beta=-0.035 ; P=0.68)$.

In the Prudent diet group, systolic blood pressure decreased by 4 (11) $\mathrm{mmHg}$ and diastolic blood pressure fell by 5 (9) $\mathrm{mmHg}$ after 2 weeks (both $P<0.02$ ) (Table 6). No significant change was observed in the Western diet group. After adjustment for baseline values, age, and sex, there was a trend towards lowered systolic blood pressure $(-3.6 \mathrm{mmHg} ; 95 \% \mathrm{CI}:-7.8$ to $0.7 ; P=0.10)$ and diastolic blood pressure $(-3.0 \mathrm{mmHg} ; 95 \% \mathrm{CI}$ : -6.2 to $0.3 ; P=0.07)$ in the Prudent diet group after 2 weeks, compared to the Western diet.

\section{Power and sample size estimates}

A secondary purpose of our pilot study was to assess the anticipated sample sizes required for detection of differences in important $\mathrm{CV}$ risk factors in a full-scale trial. These calculations for low-density lipoprotein-cholesterol (LDL-C), regional methylation of 9p21, and gene expression are presented in Additional file 3: Table S2. With 120 participants completing each intervention and control treatment period in a crossover design, with adjustment for multiple-testing of 3 pre-specified primary outcomes, we will have at least $90 \%$ power to detect a $0.75 \%$ difference in methylation of CDK2NB, and a $10 \%$ difference in 
Table 4 Nutritional Profiles of Diets Provided and Recorded as Eaten during the 2 weeks' intervention by diet assignment

\begin{tabular}{lll}
\hline Nutrient & Prudent $(n=40)$ & Western $(n=34)$ \\
\hline Energy (kcal/d) & $2162(542)$ & $1941(502)$ \\
Total protein (\% energy) & $18.9(2.9)^{\ddagger}$ & $14.4(1.7)$ \\
Available carbohydrate (\% energy) & $54.0(3.1)^{\ddagger}$ & $49.4(4.2)$ \\
Total dietary fiber (g/2000 kcal) & $37.8(5.5)^{\ddagger}$ & $12.8(5.3)$ \\
Total fat (\% energy) & $25.6(3.2)^{\ddagger}$ & $35.7(4.4)$ \\
Saturated (\% energy) & $6.3(1.6)^{\ddagger}$ & $13.9(2.4)$ \\
Polyunsaturated (\% energy) & $5.5(0.7)^{\dagger}$ & $6.2(1.6)$ \\
Monounsaturated (\% energy) & $11.6(1.5)$ & $12.0(1.7)$ \\
P:S ratio & $0.91(0.18)^{\ddagger}$ & $0.46(0.14)$ \\
Dietary cholesterol (mg/2000 kcal) & $238(90)^{\ddagger}$ & $348(68)$ \\
Alcohol (\% energy) & $0.9(1.8)$ & $0.3(0.8)$ \\
Vitamin C (mg/2000 kcal) & $256(53)^{\ddagger}$ & $79(55)$ \\
Potassium (mg/2000 kcal) & $3737(397)^{\ddagger}$ & $1864(563)$ \\
Magnesium (mg/2000 kcal) & $425(59)^{\ddagger}$ & $153(59)$ \\
Sodium (mg/2000 kcal) & $1993(355)^{\ddagger}$ & $3356(468)$ \\
Vegetables (cup eq./2000 kcal) & $3.3(0.7)^{\ddagger}$ & $1.1(0.6)$ \\
Fruits (cup eq./2000 kcal) & $3.3(0.8)^{\ddagger}$ & $0.7(0.8)$ \\
Fruits \& Veg (cup eq./2000 kcal) & $6.7(1.4)^{\ddagger}$ & $1.8(1.3)$ \\
\hline
\end{tabular}

Data are presented as means (SD). Significant between-diet differences are denoted by $\dagger$ at $P<0.05$, or $\neq$ at $P<0.01$ (independent samples t-test)

LDL-C. We will have $53 \%$ power to detect a $25 \%$ difference in gene expression.

\section{Allowance for dropout}

Similar published studies with interventions of this type and duration have reported dropout rates of $<10 \%[17,18]$. Therefore, the full scale study would need to enroll 110 participants per arm. Imputing a change value of "0" for an upper limit of $10 \%$ who dropout, our estimated power will be $86 \%$ for each of the methylation, LDL-C, and gene expression outcomes.

\section{Discussion}

This 'proof of concept' randomized controlled feeding trial confirmed that recruitment of apparently healthy individuals into a study that provides either a Prudent or Western diet is feasible and retention is high. Both diets were reported to be palatable, well tolerated, and small changes in cardiovascular risk factors (which trended towards statistical significance) and body composition were seen over a short time period in the Prudent diet.

We were able to recruit our study population over eight months, through newspaper advertisements, campus posters, and referrals. During conversations with participants, the two strongest reasons for participation were provision of food, and the chance to learn about their genetic risk for cardiovascular disease. Given our recruitment catchment area (a university campus and surrounding area) and target population - young, healthy men and women-it was not surprising that food provision was an attractive incentive for participation. However, the majority of participants expressed an interest in knowing their genetic risk for cardiovascular disease, and gaining a better understanding of how to change their lifestyles to modify this risk-suggesting that in a highly-educated catchment area, health education (and possibly "novel" health education) is an attractive feature of research study participation. In our study, participant retention was excellent and self-reported adherence to the diet plans was high, which we credit to the short duration of the intervention, and offering participants flexible options with respect to how they would obtain their foods for the study.

A first major design consideration was determining the macro and micronutrient composition of the "Western" and "Prudent" diets. We attempted to model the "Western" diet after the typical Canadian macronutrient profile [19], while retaining elements of well-studied "Western" dietary patterns [15]. The Western diet contained more saturated fat and less polyunsaturated fat, less fibre, and

Table 5 Diet palatability, satiety, and gastrointestinal symptoms experienced on Prudent and Western diets

\begin{tabular}{|c|c|c|c|c|c|c|c|}
\hline & \multicolumn{3}{|c|}{ Prudent diet ( $n=33$ to 45$)$} & \multicolumn{3}{|c|}{ Western diet ( $n=31$ to 45$)$} & \multirow{2}{*}{$\begin{array}{l}\text { Mean difference between Prudent and } \\
\text { Western }(95 \% \mathrm{Cl}) \text { at } 2 \text { weeks }\end{array}$} \\
\hline & Week 0 & Week 1 & Week 2 & Week 0 & Week 1 & Week 2 & \\
\hline Palatability, mean (SD) (out of 10$)^{a}$ & $8.2(1.5)$ & $6.9(1.3)$ & $7.1(1.4)$ & $7.9(1.3)$ & $4.6(1.7)$ & $4.8(1.4)$ & $2.2(1.6 \text { to } 2.9)^{\text {b§ }}$ \\
\hline Satiety $(-3$ to +3$)$ & $1.1(1.1)$ & $1.3(1.5)$ & $1.2(1.1)$ & $0.7(1.3)$ & $1.0(1.8)$ & $0.9(1.2)$ & $0.3(-0.3 \text { to } 0.9)^{b}$ \\
\hline \multicolumn{8}{|l|}{ Gas/bloating, n (\%) } \\
\hline As usual or less than usual & $32(86.5)$ & $26(76.5)$ & $25(75.8)$ & $31(91.2)$ & $22(62.9)$ & $17(54.8)$ & $0.047^{c}$ \\
\hline More than usual & $5(13.5)$ & $8(23.5)$ & $8(24.2)$ & $3(8.8)$ & $13(37.1)$ & $14(45.2)$ & \\
\hline \# bowel movements per week, mean (SD) & $7.6(5.6)$ & $7.5(6.2)$ & $7.4(6.1)$ & $6.8(4.4)$ & $7.0(4.2)$ & $6.5(4.7)$ & $0.4(-1.5 \text { to } 2.2)^{b}$ \\
\hline Any days of diarrhea, $n(\%)$ & $4(4.9)$ & $4(4.9)$ & $3(3.7)$ & $1(1.2)$ & $3(3.7)$ & $4(4.9)$ & $>0.70^{c}$ \\
\hline Any days of constipation, $n(\%)$ & $5(6.1)$ & $4(4.9)$ & $1(1.2)$ & $2(2.4)$ & $12(14.6)$ & $9(11.0)$ & $<0.001^{c}$ \\
\hline
\end{tabular}

${ }^{a}$ Week 0 is palatability of their usual diet while weeks 1 and 2 reflect the palatability of the intervention diet

${ }^{\mathrm{b}}$ Comparison of mean intervention values (week 1 and 2), adjusted for age, sex, and baseline values. Expressed as mean $(95 \% \mathrm{Cl})$; ${ }^{\dagger} P<0.05,{ }^{\ddagger} P<0.01,{ }^{5} P<0.001$ 'Fisher's exact test for between-treatment differences in distribution of variables during follow up. Counts of number of participants reporting any days of diarrhea, constipation, or more bloating/gas in either week 1 or 2 were collapsed for analysis, if such data were also provided at baseline 
Table 6 Effects of Prudent and Western diets on body weight, blood pressure, and lipid risk factors

\begin{tabular}{|c|c|c|c|c|c|c|c|}
\hline & \multicolumn{3}{|c|}{ Prudent $(n=45)$} & \multicolumn{3}{|c|}{ Western $(n=37)^{*}$} & \multirow[b]{2}{*}{ Prudent minus Western ${ }^{\mathrm{b}}$} \\
\hline & Week $0^{\mathrm{a}}$ & Week $2^{\text {a }}$ & Change $^{a}$ & Week $0^{\mathrm{a}}$ & Week $2^{\mathrm{a}}$ & Change $^{a}$ & \\
\hline Body weight & $72.6(16.4)$ & $72.0(16.1)$ & $-0.6(1.4)^{\ddagger}$ & $69.3(13.3)$ & $68.9(13.0)$ & $-0.4(1.2)^{\dagger}$ & $-0.1(-0.7$ to 0.4$)$ \\
\hline Body-mass index $\left(\mathrm{kg} / \mathrm{m}^{2}\right)$ & $26.1(5.6)$ & $25.9(5.4)$ & $-0.1(1.3)$ & $25.1(4.6)$ & $25.0(4.4)$ & $-0.1(0.4)$ & $-0.02(-0.44$ to 0.40$)$ \\
\hline Percent body fat & $27.9(9.0)$ & $28.2(8.5)$ & $-1.3(3.2)^{\ddagger}$ & $28.6(11.7)$ & $29.9(11.3)$ & $0.3(3.3)$ & $-1.5(-2.9$ to -0.04$)$ \\
\hline \multicolumn{8}{|l|}{ Blood pressure $(\mathrm{mmHg})$} \\
\hline Systolic & $118(17)$ & $114(16)$ & $-4(11)^{\dagger}$ & $112(18)$ & $113(15)$ & $2(10)$ & $-4(-8$ to 1$)$ \\
\hline Diastolic & $74(10)$ & $70(10)$ & $-5(9)^{\S}$ & $74(10)$ & $72(9)$ & $-1(6)$ & $-3(-6$ to 0.3$)$ \\
\hline \multicolumn{8}{|l|}{ Lipids (mmol/L) } \\
\hline Total-Cholesterol & $4.92(1.26)$ & $4.73(1.24)$ & $-0.19(0.65)^{\dagger}$ & $5.25(1.05)$ & $5.23(1.14)$ & $0.03(0.51)$ & $-0.24(-0.51$ to 0.03$)$ \\
\hline Non-HDL-C & $3.43(1.14)$ & $3.29(1.13)$ & $-0.14(0.64)$ & $3.81(0.94)$ & $3.73(0.99)$ & $-0.03(0.55)$ & $-0.15(-0.42$ to 0.12$)$ \\
\hline
\end{tabular}

Data are presented as ${ }^{a}$ Mean (SD) or ${ }^{b}$ mean $(95 \% \mathrm{Cl})$; ${ }^{*}$ Significance of between-treatment difference in parameter was estimated using analysis of covariance, adjusted for age, sex, and baseline values. Significant between-diet differences are denoted by ${ }^{\dagger}(P<0.05),{ }^{\ddagger}(P<0.01)$, or ${ }^{\S}(P<0.001)$

more cholesterol than the Prudent diet. The Western diet was also designed to be lower in vitamin $\mathrm{C}$ and potassium, and higher in sodium than the Prudent diet. No specific targets were set for protein and total carbohydrate, but as a function of the foods chosen, the Prudent diet was higher in protein and total carbohydrate.

A second major design consideration was how to translate the diets into viable meal options. To do this, we constructed the "Western" diet around highly processed foods; and the "Prudent" diet around minimally processed foods. The resulting nutrient composition was indicative of an important difference in diet quality. In the "Western" diet, protein sources were meats such as beef burgers, fried chicken, and higher-fat deli meats; carbohydrate sources were refined grains, cereals, and sweets such as white bread, corn flakes, jelly beans; fat sources were full-fat dairy, processed cheeses, and butter. Minimal fruits and vegetables were included, and these were mostly processed items, such as juices and French fries. In the Prudent diet, protein sources were lean meats (e.g., chicken breast), fish (e.g., salmon fillets), and legumes (e.g., bean salads); carbohydrate sources were whole grains and fruits such as brown rice, steel-cut oats, and bananas; fat sources were plant oils such as canola and olive oils (used in dressings for salads/cooking) and fish oils occurring in salmon and tuna. A high amount of fresh fruits and vegetables were provided. People were encouraged to use the provided cooking fats for preparation of meals (e.g. butter for Western; unsaturated oils for Prudent). Cooking suggestions with meal plans were provided, but flexibility was given so that the participants could prepare the foods as they wished.

A third consideration for this pilot study was determining the optimal length of the intervention. We chose 2 weeks, as previous reports have shown that changes in cardiovascular risk factors such as lipids and blood pressure in response to diet modification can be observed within this time frame [9-12]. Additionally, others have demonstrated that epigenetic changes in response to dietary modification can be observed in as little as 5 days [20]. With these considerations, we deliberately designed the two diets to be very different (i.e. on extreme ends of the "healthy eating" spectrum). This between-diet "healthfulness contrast" was intended to maximize our ability to see changes in a short time period in apparently healthy individuals. Furthermore, we had serious reservations about placing study volunteers on a dietary pattern known to be detrimental to health (i.e. the "Western" dietary pattern) for longer than 2 weeks. However, feeding trials which have substantially impacted public health policy have employed 4- to 6-week feeding periods [12, 21, 22] and in the planned future trial we will extend our treatment period to 4 weeks. A limitation of this short duration was that we found it difficult to precisely calibrate prescribed food intake to match energy expenditure, which resulted in small, but significant weight loss within both diet groups. In our full-scale trial, we intend to incorporate a longer run-in period to obtain better estimates of maintenance energy requirements that incorporate anthropometric characteristics as well as participants' usual energy intakes.

A fourth consideration was whether to provide food for participants or provide counseling only. We felt that food provision was the best way to ensure high adherence to the dietary principles, but our design fell short of the conventional approach to a controlled feeding study in a few respects. We were not able to admit participants for a hospital stay or metabolic ward for the duration of the study [23], we could not provide cooked meals for participants [24], and we did not have the facilities to have participants eat at least 1 meal per day on site [22]. Notwithstanding these challenges, provision of food without observation of participant eating occasions or inpatient admission has been shown to result in high adherence to dietary advice in settings similar to ours [21]. 
Participant feedback on the implementation of the meal plans will be most useful in planning the full trial. First, though food provision was viewed positively by participants, the usefulness of this could have been greatly enhanced if more careful attention was paid to designing attractive menu items, rather than simple suggestions of which foods to eat at specified times/eating occasions.

Second, both diets posed challenging for participants to consume, but for different reasons. The volume of the foods on the whole-foods, plant-based Prudent diet was difficult to consume for many participants, and this was reflected in slightly but significantly lower adherence in this arm-i.e. less of the prescribed foods were eaten (e.g. smaller portion sizes of vegetables) or some foods were omitted (e.g. snacks). The "Western" diet was a marked departure from usual eating habits for most people, which we did expect. To help mitigate the anticipated dissatisfaction with the "Western" diet, we offered diet counseling with a registered dietitian to all participants free-of-charge after study completion to help improve their eating habits. Only one participant took advantage of this offer.

Third, the preparation time of foods on the Prudent diet was noted as a major inconvenience for busy students. While this might be viewed as an expected outcome of a whole-foods approach to eating, for the purposes of participant satisfaction and retention, modifications to the meal plans may be considered to make the meals easier to prepare and consume for working/student participants. These concerns can be addressed in a full-scale trial by seeking input from not only a dietitian but also a chef to plan meal suggestions.

Fourth, we learned that the incorporation of some flexibility into our meal plans will aid adherence, especially in a full-scale trial of longer duration. In addition to relatively minor substitutions of food items that can be made without impacting the spirit of the intended diets (e.g. lean beef for poultry; fast-food turkey submarine for deli meat sandwiches; fruit or nut exchanges, etc.), menu plans designed for vegetarian participants or those with aversions to dairy would increase the number of eligible participants.

The strengths of our study include (i) full food provision, which enhanced adherence and allowed for a large contrast in dietary profiles; (ii) the collection of biological samples for biochemical, clinical, genetic, and epigenetic assessment at baseline and post-feeding; and (iii) the choice to have food delivered using any one of three delivery channels. The limitations are that (i) the study population was small, (ii) the duration of the intervention was short (2-weeks), (iii) we cannot verify that the food provided to the participants was actually consumed by the individual enrolled in the study (versus being thrown away or eaten by others in the household), and (iv) we did not have the facilities to provide any meals on-campus, as is often done in most feeding studies.

\section{Conclusions}

The recruitment of and provision of food to apparently healthy adults for the purpose of a 2-week intervention outside of a specialty clinic was well received and had high retention. A definitive trial powered to detect epigenetic changes would be feasible in a similar population, with some adaptations to the protocol.

\section{Additional files}

Additional file 1: Table S1. Representative menu plans for the Western and prudent diets. (DOCX $62 \mathrm{~kb}$ )

Additional file 2: Table S3. Participant feedback provided with respect to each diet. (DOCX $13 \mathrm{~kb}$ )

Additional file 3: Table S2. Sample size calculations for a cross-over trial $[25,26]$. (DOCX $113 \mathrm{~kb})$

\section{Abbreviations \\ ANRIL, antisense non-coding RNA in the INK4 locus; BMI, body-mass index; CAD, Canadian dollars; CDKN2A, cylcin-dependent kinase inhibitor 2A; CDKN2B, cylcin-dependent kinase inhibitor 2B; CRCTL, Clinical Research and Clinical Trials Laboratory; CV, cardiovascular; CVD, cardiovascular disease; DIGEST, Diet and Genetic Intervention Study; DNA, Deoxyribonucleic acid; HDL-C, high-density lipoprotein cholesterol; Kcal, kilocalories (of food energy); LDL-C, low-density lipoprotein; MI, myocardial infarction; P:S, Polyunsaturated: saturated fat ratio (\% energy/\% energy); SD, standard deviation; TC, total cholesterol; TG, triglycerides}

\section{Acknowledgements}

We are indebted to our participants for their commitment to the study. This study was funded by a grant from the Labarge Healthy Aging Initiative to Sonia S. Anand. The funding agency had no role in the design of the study, data collection, analysis, or interpretation of data. The funding agency had no role in the writing of the manuscript; or the decision to submit the manuscript for publication.

\section{Availability of data and materials}

Because sensitive genetic material was collected as part of this pilot study, data from this study will not be shared.

\section{Authors' contributions}

Andrew Mente and Sonia S. Anand conceptualized the study. Russell J. de Souza, Andrew Mente, Sujane Kandasamy, Dipika Desai, Guillaume Pare, Joseph Beyene and Sonia S. Anand designed the study. Russell J. de Souza, Andrew Mente, Sujane Kandasamy, Monisha Nundy, and Dipika Desai acquired the data. Michael A. Zulyniak, Russell J. de Souza, Kripa Raman, and Ranya Hasso analyzed the data. Michael A. Zulyniak, Russell J. de Souza, Andrew Mente, Guillaume Pare, Joseph Beyene, and Sonia S. Anand contributed to interpretation of the data. Michael A. Zulyniak and Russell J. de Souza drafted the manuscript. Andrew Mente, Sujane Kandasamy, Dipika Desai, Ranya Hasso, Guillaume Pare, Joseph Beyene, and Sonia S. Anand critically revised the manuscript for important intellectual content. All authors have given final approval of this version of the submitted manuscript, and agree to be accountable for all aspects of the work in ensuring that questions related to the accuracy or integrity of any part of the work are appropriately investigated and resolved.

\section{Competing interests}

During the time that this study was conducted, Russell J. de Souza held a Canadian Institutes for Health Research (CIHR) postdoctoral fellowship and 
Michael A. Zulyniak held a CIHR Randomized Controlled Trials Mentoring grant. All other authors declare no competing interests.

\section{Consent for publication}

This manuscript does not contain any individual person's data.

\section{Ethics approval and consent to participate}

The study protocol was submitted to the Hamilton Integrated Research Ethics Board (HiREB) on April 20th, 2012 and granted final approval on May 24th, 2012 (\#12-260). The trial was registered at clinicaltrials.gov (Trial Registration: NCT01658137). All participants provided informed consent to participate in this research study.

\section{Author details}

${ }^{1}$ Department of Medicine, McMaster University, Hamilton, Ontario, Canada. ${ }^{2}$ Department of Clinical Epidemiology and Biostatistics, McMaster University, Hamilton, Ontario, Canada. ${ }^{3}$ Department of Nutritional Sciences, University of Toronto, Toronto, Ontario, Canada. ${ }^{4}$ Li Ka Shing Knowledge Institute, St. Michael's Hospital, Toronto, Ontario, Canada. ${ }^{5}$ Population Health Research Institute, Hamilton Health Sciences and McMaster University, Hamilton, Ontario, Canada.

\section{Received: 18 March 2016 Accepted: 4 June 2016}

Published online: 10 June 2016

\section{References}

1. Heart and Stroke Foundation of Canada. Statistics - Heart and Stroke Foundation. 2013 [cited 2014 08-2014]; Available from: http://www. heartandstroke.com/site/c.ikIQLcMWJtE/b.3483991/k.34A8/Statistics.htm.

2. O'Donnell CJ, Nabel EG. Genomics of cardiovascular disease. N Engl J Med. 2011;365(22):2098-109.

3. Kessler T, Erdmann J, Schunkert H. Genetics of coronary artery disease and myocardial infarction-2013. Curr Cardiol Rep. 2013;15(6):1-8.

4. McPherson $\mathrm{R}$ et al. A common allele on chromosome 9 associated with coronary heart disease. Science. 2007;316(5830):1488-91.

5. Palomaki GE, Melillo S, Bradley LA. Association between 9p21 genomic markers and heart disease: a meta-analysis. Jama. 2010;303(7):648-56.

6. Cunnington MS, Keavney B. Genetic mechanisms mediating atherosclerosis susceptibility at the chromosome 9p21 locus. Curr Atheroscler Rep. 2011; 13(3):193-201.

7. Holdt LM, Teupser D. Recent studies of the human chromosome 9p21 locus, which is associated with atherosclerosis in human populations. Arterioscler, Thromb, Vasc Biol. 2012;32(2):196-206.

8. Do $R$ et al. The effect of chromosome 9 p21 variants on cardiovascular disease may be modified by dietary intake: evidence from a case/control and a prospective study. PLoS Med. 2011;8(10):e1001106.

9. Jenkins DJ et al. Effect of a diet high in vegetables, fruit, and nuts on serum lipids. Metabolism. 1997;46(5):530-7.

10. Hodson L, Skeaff CM, Chisholm WA. The effect of replacing dietary saturated fat with polyunsaturated or monounsaturated fat on plasma lipids in free-living young adults. Eur J Clin Nutr. 2001;55(10):908-15.

11. Brancati FL et al. Effect of potassium supplementation on blood pressure in African Americans on a low-potassium diet. A randomized, double-blind, placebo-controlled trial. Arch Intern Med. 1996;156(1):61-7.

12. Appel $\sqcup$ et al. A clinical trial of the effects of dietary patterns on blood pressure DASH Collaborative Research Group. N Engl J Med. 1997;336(16):1117-24.

13. Jenkins DJ et al. Effect of legumes as part of a low glycemic index diet on glycemic control and cardiovascular risk factors in type 2 diabetes mellitus: a randomized controlled trial. Arch Intern Med. 2012;172(21):1653-60.

14. Karanja NM et al. Descriptive characteristics of the dietary patterns used in the Dietary Approaches to Stop Hypertension Trial. DASH Collaborative Research Group. J Am Diet Assoc. 1999;99(8 Suppl):S19-27.

15. Fung $T T$ et al. Association between dietary patterns and plasma biomarkers of obesity and cardiovascular disease risk. Am J Clin Nutr. 2001;73(1):61-7.

16. Kien $\mathrm{CL}$, Ugrasbul F. Prediction of daily energy expenditure during a feeding trial using measurements of resting energy expenditure, fat-free mass, or Harris-Benedict equations. Am J Clin Nutr. 2004;80(4):876-80.

17. Kris-Etherton PM, Dietschy J. Design criteria for studies examining individual fatty acid effects on cardiovascular disease risk factors: human and animal studies. Am J Clin Nutr. 1997;65(5 Suppl):1590S-6S.
18. Christensen AS et al. Effect of fruit restriction on glycemic control in patients with type 2 diabetes-a randomized trial. Nutr J. 2013;12:29.

19. Health Canada. Canadian Community Health Survey, Cycle 2.2, Nutrition (2004)- Nutrient Intakes from Food: Provincial, Regional, and National Summary Data Tables. H. Canada, Editor. Ottawa, ON: Publications, Health Canada; 2008.p. 224.

20. Jacobsen SC et al. Young men with low birthweight exhibit decreased plasticity of genome-wide muscle DNA methylation by high-fat overfeeding. Diabetologia. 2014;57(6):1154-8.

21. Jenkins DJ et al. Effects of a dietary portfolio of cholesterol-lowering foods vs lovastatin on serum lipids and C-reactive protein. JAMA. 2003;290(4):502-10.

22. Carey VJ et al. Rationale and design of the Optimal Macro-Nutrient Intake Heart Trial to Prevent Heart Disease (OMNI-Heart). Clin Trials. 2005;2(6):529-37.

23. Mukuddem-Petersen $J$ et al. Effects of a high walnut and high cashew nut diet on selected markers of the metabolic syndrome: a controlled feeding trial. Br J Nutr. 2007;97(6):1144-53.

24. Swain JF et al. Menu design and selection for multicenter controlled feeding studies: process used in the Dietary Approaches to Stop Hypertension trial. DASH Collaborative Research Group. J Am Diet Assoc. 1999;99(8 Suppl):S54-9.

25. Holdt LM et al. ANRIL expression is associated with atherosclerosis risk at chromosome 9p21. Arterioscler, Thromb, Vasc Biol. 2010;30(3):620-7.

26. Obarzanek $E$ et al. Effects on blood lipids of a blood pressure-lowering diet: the Dietary Approaches to Stop Hypertension (DASH) Trial. Am J Clin Nutr. 2001;74(1):80-9.

\section{Submit your next manuscript to BioMed Central and we will help you at every step:}

- We accept pre-submission inquiries

- Our selector tool helps you to find the most relevant journal

- We provide round the clock customer support

- Convenient online submission

- Thorough peer review

- Inclusion in PubMed and all major indexing services

- Maximum visibility for your research

Submit your manuscript at www.biomedcentral.com/submit 